\title{
Action-specific perception of speed is independent of attention
}

\author{
Jessica K. Witt ${ }^{1} \cdot$ Mila Sugovic $^{2} \cdot$ Michael D. Dodd ${ }^{3}$
}

Published online: 12 January 2016

(C) The Psychonomic Society, Inc. 2016

\begin{abstract}
According to the action-specific account of perception, a perceiver's ability to act influences how the environment is perceived. For example, in a computer-based task, participants perceive fish as moving faster when they use a smaller net, and are thus less effective at catching the fish (Witt \& Sugovic, 2013a). Here, we examined the degree to which attention may influence perceptual judgments by requiring participants to engage in a secondary task that directed their attention either toward (Exp. 1) or away from (Exp. 2) the to-be-caught fish. Though perceived fish speed was influenced by participants' catching performance-replicating previous results - attentional allocation did not impact this relationship between catching performance and perceived fish speed. The present results suggest that action directly influences spatial perception, rather than exerting indirect effects via attentional processes.
\end{abstract}

Keywords Spatial perception · Embodied perception · Perception-action coupling $\cdot$ Attention $\cdot$ Affordances

People perceive the spatial layout of the environment in relation to their ability to act. For example, softball players who are hitting better than others see the ball as bigger (Witt \&

Jessica K. Witt

jessica.witt@colostate.edu

1 Department of Psychology, Colorado State University, Fort Collins, CO 80523, USA

2 Department of Psychological Sciences, Purdue University, West Lafayette, IN, USA

3 Department of Psychology, University of Nebraska, Lincoln, NE, USA
Proffitt, 2005). Archers who are shooting better than others see the target as bigger (Lee, Lee, Carello, \& Turvey, 2012). Athletes trained in parkour see walls as being shorter than do untrained novices (Taylor, Witt, \& Sugovic, 2011). Weighted objects are perceived to be lighter when the perceiver anticipates carrying the object with the help of a capable partner, relative to when the object must be carried alone or with an unable partner (Doerrfeld, Sebanz, \& Shiffrar, 2012). When an individual's ability to interact with objects in the environment changes the manner in which objects are perceived, this is considered an action-specific effect (e.g., Witt, 2011). Action-specific effects have been documented in a wide range of experimental contexts, including in laboratory and natural settings, with experimental manipulations and naturally occurring differences, and with direct, indirect, and implicit measures.

The action-specific account of perception has its roots in Gibson's (1979) theory of affordances, with affordances being opportunities for action within the environment. Actionspecific effects demonstrate that affordances are perceived even when making judgments regarding spatial aspects of the environment such as distance, size, and speed. To truly understand the theoretical implications of action-specific effects, however, the underlying mechanism(s) must be determined. For instance, if affordances are so fundamental that the perceiver cannot help but implicitly report them, this would be strong support for the idea that affordances are the primary objects of perception (Lee et al., 2012).

It is also possible that action-specific effects are attributable to preperceptual processes related to attention. According to an attention-based mechanism, a target object appears bigger, slower, farther, or steeper under one action condition because perceivers attend to the target differentially as a function of the task they intend to perform. An attention-based mechanism is intuitively appealing, given that it is already known that action 
requirements influence how perceivers allocate attention (Hayhoe \& Ballard, 2005), and that the allocation of attention influences action-related performance (e.g., Vickers, 1992). Moreover, current theories of spatial perception can readily accommodate action-specific effects that are due to attention (Philbeck \& Witt, 2015; Witt \& Riley, 2014). For example, many theories consider distance perception to be the product of weighting various optical cues for depth such as binocular disparity and depth from motion (e.g., Johnston, Cumming, \& Landy, 1995). Changes in attention due to action could impact either the cues that are selected for perceptual integration or the weights given to each cue without action directly impacting perception (Philbeck \& Witt, 2015).

Attention as an underlying mechanism could have further theoretical implications, however, in that theorists may discount action-specific effects as being perceptual (Firestone $\&$ Scholl, 2015). In challenging whether action-specific effects are truly perceptual, Firestone and Scholl have put forth six pitfalls that account for all recent claims of top-down effects on perception, including action-specific effects. These pitfalls need not apply in every case, but if just one pitfall can account for the results, the effect should be discounted as being a genuine influence on perception. With respect to the paradigm used in the present article (described below), previous research has examined five of the six pitfalls and found that none can account for this effect (Witt, Sugovic, Tenhundfeld, \& King, 2015). Thus, the one remaining pitfall relates to attentional allocation. Firestone and Scholl argue that purported perceptual biases such as action-specific effects should not be considered genuine if they can be explained by differences in attention. The goal of the present study was to determine whether attention drives action-specific effects. To do this, we explicitly manipulated the requirement to directly attend —or not attend - a to-be-acted-upon target.

Numerous researchers have proposed that attention may be the critical factor underlying action-specific effects (Bloesch, Davoli, Roth, Brockmole, \& Abrams, 2012; Cañal-Bruland, Pijpers, \& Oudejans, 2010; Cañal-Bruland \& van der Kamp, 2009; Cañal-Bruland, Zhu, van der Kamp, \& Masters, 2011; Gray, 2013; Gray, Navia, \& Allsop, 2014). For example, despite not explicitly examining/manipulating attention, Bloesch and colleagues (2012) posited that attention can account for why targets look closer when one observes someone else reach with a tool, relative to observing that same person reach without the tool. Similarly, in a flight simulator, Gray and colleagues (2014) observed that both airplane-landing performance and time spent fixating the runway correlated with judgments of runway size: Individuals who performed the landing more accurately and/or fixated the runway longer judged the runway to be bigger. These authors argued for an attention-based mechanism on the basis of pair-wise correlations, but the complete relationship between fixations, performance, and perceived size was not reported. To this point, therefore, the evidence favoring a critical role for attention has been mostly indirect. Additionally, fixing attentional allocation to a particular location does not diminish the effect of apparent body size on the perceived size of a nearby object (van der Hoort \& Ehrsson, 2014).

Other researchers have also argued for a role of attention, but they have treated it as a boundary condition rather than an underlying mechanism. For example, Cañal-Bruland and colleagues (2011) reported that golf-putting performance correlated with the perceived size of the golf hole when attention could be directed toward the hole, but not when attention was obscured or diverted away from the hole. They advanced a perceptual accentuation hypothesis that rests on the notion that attention must be directed toward an object in order for the object to be perceived in terms of action. The perceptual accentuation hypothesis does not consider attention to be a mediating factor; that is, differences in attention across various action conditions do not drive action-specific effects, but rather, attention acts as a moderator or a boundary condition. Attention is necessary and sets the stage for action-specific effects, according to this account, but it does not account for the effects themselves.

Taken together, the evidence favoring a role for attention in action-specific effects is decidedly mixed, with some evidence existing for each of three existing hypotheses, all of which make separate predictions. According to an attention-based account, individual or experimental variations in one's ability to act lead to differences in the allocation of attention, which produce differences in the perception of the target. Thus, any manipulation that can ensure that attention is equated across conditions should lead to an elimination of action-specific effects. According to a perceptual accentuation hypothesis, attention is necessary but not sufficient to explain action-specific effects. Here, the key prediction is that diverting attention away from the target - rather than equating attention across conditions - should eliminate action-specific effects. According to non-attention-based accounts, action-specific effects should persist despite any variations in attention, including either equating attention across conditions or diverting attention away from the target. In the present study, we explicitly tested these three accounts via direct manipulations of attention.

We examined the role of attention in the context of the effect of catching ability on apparent speed. In this paradigm, participants attempted to catch a fish moving across a computer screen by shooting a net toward the fish. The net varied in size, which influenced the ease with which the fish could be caught. When the net was bigger - meaning that the task of catching the fish was easier-participants perceived the fish as moving slower than when the net was smaller and the task was more difficult (Witt \& Sugovic, 2013a). This paradigm was modeled after a similar paradigm in which participants attempted to catch a moving ball by continuously controlling the vertical position of a paddle. In the ball- 
catching paradigm, participants perceived the ball as moving slower when the paddle was bigger, and more effective, than when the paddle was smaller (Witt \& Sugovic, 2010, 2012).

This action-specific effect of catching ease on perceived speed affords an opportunity to examine the influence of attention, given the overwhelming evidence that net (or paddle) size influences perceived fish (or ball) speed, as opposed to postperceptual processes such as memory or task demands (Witt, South, \& Sugovic, 2014; Witt \& Sugovic, 2010, 2012, 2013a, b, c; Witt, Sugovic, \& Taylor, 2012), thus ruling out Pitfalls \#2 (judgment vs. perception), \#3 (task demands), and \#6 (memory vs. perception). The results also showed that these effects are due to the ease of catching the target, rather than to visual differences across net sizes or individual trial outcomes, thus ruling out Pitfalls \#1 (overly confirmatory research) and \#4 (low-level differences). For example, the effect of net or paddle size on speed judgments is also found when judgments are made while the fish (or ball) is still moving (Witt \& Sugovic, 2012, 2013a), when the analyses control for compliancy with task demands (Witt \& Sugovic, 2013b), and when the perceptual measure is implicit and action-based (Witt \& Sugovic, 2013a). Having ruled out five of the six pitfalls (Witt et al., 2015), the next order of business is to address the final pitfall.

Some insight into the manner in which attention could influence perception in the present task can be gleaned from the Aubert-Fleischl illusion, in which a moving object appears slower when an observer tracks it, relative to when a nearby stationary point is fixated (Aubert, 1886; Fleischl, 1882). Importantly for the present purposes, attentional allocation directly influences the perceived speed of the object. Therefore, if net size gives rise to differences in attentional allocation, this could explain why the fish appears to move at different speeds across different net sizes. Specifically, if perceivers attend to the fish more when the net is big than when the net is small, this would explain why the fish appears slower when the participant plays with a bigger net.

In the present experiments, we sought to delineate between the attention-based, perceptual accentuation, and nonattention-based accounts of action-specific effects. To that end, we employed a secondary task that could only be performed by continuously attending the target fish (Exp. 1) or by continuously diverting attention away from the target fish (Exp. 2). If differential attentional allocation is critical, then we would not expect to observe action-specific effects in Experiment 1 , given that the secondary task should equate the levels of engagement with the target fish independent of net size and fish speed. If attention instead is an important moderating variable, then we would expect a reduction or elimination of action-specific effects in Experiment 2, when attention was diverted away from the target. If, however, attention does not play a critical role, then neither manipulation should impact the presence or magnitude of action-specific effects.

\section{Experiment 1: focusing attention on the target}

Participants attempted to block a fish moving across the screen, while using different-sized nets. Participants pressed the trigger on a joystick to release the net, which then moved upward on the computer screen perpendicular to the horizontal movement of the fish. If the net intersected the fish, the catch was successful; otherwise, the fish continued past the net, and the trial was a miss. To be successful, participants had to accurately time the release of the net just right. After each attempt, participants estimated the speed of the fish.

To control attentional allocation, we employed a secondary task in which participants were required to count the number of times that a small square briefly appeared on the fish. The square was small, and thus was designed to force participants to focus on the fish and not to look elsewhere. This manipulation should ensure that attention was continuously allocated toward the fish in all conditions, independent of net size and fish speed.

\section{Method}

Participants A total of 27 volunteers provided informed consent and participated in exchange for course credit. All participants were naïve as to the purpose of the study, which took place in a single 30-min session. A post-hoc power analysis indicated that we achieved over $95 \%$ power with this number of participants to obtain the effect of net size on perceived fish speed.

Stimuli and apparatus Stimuli were presented on a 19-in. desktop monitor with a black background (see Fig. 1). A white fish, $1.9 \mathrm{~cm}$ wide and $1.8 \mathrm{~cm}$ tall, was the target. The net was a white rectangle that was $0.7 \mathrm{~cm}$ wide and was set to one of three heights on each trial $(1.97,5.92$, or $11.84 \mathrm{~cm})$. Participants controlled the release of the net with a joystick. The square was black, $0.38 \mathrm{~cm}^{2}$ in size, and was presented at the center of the fish.

Procedure Participants were initially trained on the slow $(18 \mathrm{~cm} / \mathrm{s})$ and fast $(74 \mathrm{~cm} / \mathrm{s})$ anchor speeds. During this initial training, text on the screen indicated which anchor speed would be shown prior to each exposure. Each anchor speed was shown three times, and order was randomized. Next, the anchor speeds were shown again but were not labeled, and participants had to identify each speed as "slow" or "fast" by pressing the corresponding button on the joystick. The speeds were shown three times each, order was randomized, and text on the screen ("Correct" in green or "Incorrect" in red) gave participants feedback on their responses.

After training, participants were instructed how to perform the task. They were told that their task was to catch the fish by pressing the trigger on the joystick to release the net. After 


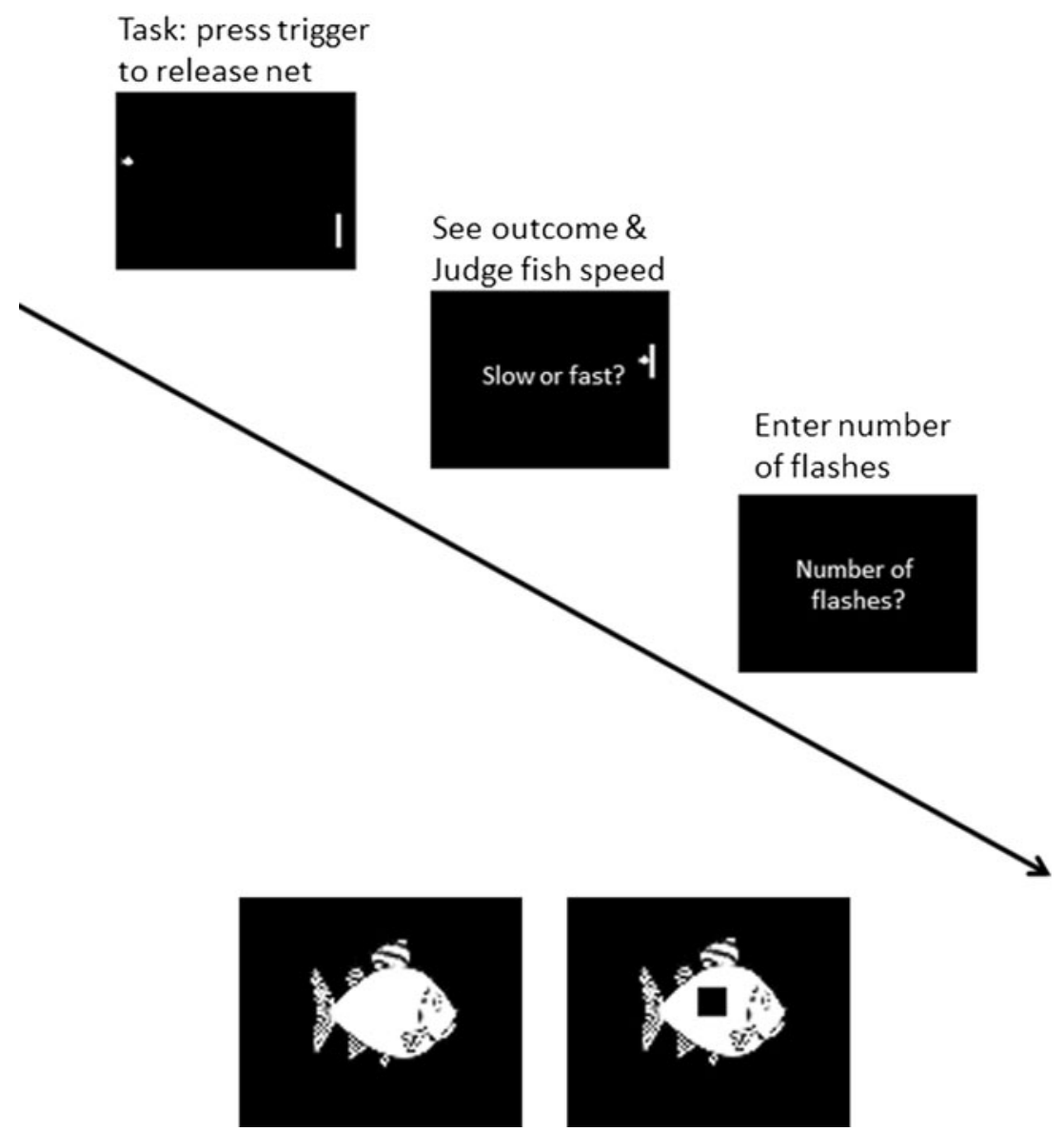

Fig. 1 Overview of the experimental task (top). On this trial, the medium net is depicted, and the trial resulted in a successful catch. Here, the text on the screen is shown bigger in order to be legible. Also shown are close-ups of the fish with the square absent and present (bottom)

each attempt to catch the fish, they were asked to perform a speed bisection task (e.g., Witt \& Sugovic, 2010), by indicating whether the fish moved more like the slow or the fast training speed via buttonpress on the joystick. Participants were also told that a small black square would flash on the center of the fish at various times (see Fig. 1). Their task was to count the number of times the square flashed ${ }^{1}$ and to enter this number into the keyboard at the end of the trial. This represented the critical secondary task that required continuous attention to the fish in order to be performed correctly.

On each trial, the fish and the net appeared for $50 \mathrm{~ms}$ before the fish moved across the screen. The fish was positioned either high $(21.4 \mathrm{~cm}$ from the bottom of the monitor) or low (17.5 $\mathrm{cm}$ from the bottom). The fish moved from left to right at one of six speeds, ranging from 18 to $65 \mathrm{~cm} / \mathrm{s}(18.46,27.69$, $36.91,46.14,55.37$, or $64.60 \mathrm{~cm} / \mathrm{s}$ ). The center of the net was positioned $6.5 \mathrm{~cm}$ from the bottom and $2.4 \mathrm{~cm}$ from the right

\footnotetext{
${ }^{1}$ The term "flash" was used when instructing participants; however, it is critical to note that the appearance and subsequent disappearance of the square on the fish was subtle and could only be detected via sustained attention to the fish. This was not a flash or an onset of the type that would lead to the automatic capture of attention via exogenous cues.
}

edge of the monitor. Upon pressing the joystick trigger, the net moved up at a constant speed of $18 \mathrm{~cm} / \mathrm{s}$. If any part of the front edge of the net intersected any part of the fish, both the fish and net stopped immediately. These were classified as successful catching trials. Otherwise, both objects continued until the fish was beyond the edge of the screen, and the trial was classified as a miss.

The square appeared at random at the center of the fish while the fish moved across the screen. When the square appeared, it stayed visible for $80 \mathrm{~ms}$ before disappearing; it could not appear again within $120 \mathrm{~ms}$ of the previous appearance. Once the fish had moved within $2.4 \mathrm{~cm}$ of the net, the square could no longer appear. The maximum number of times that the square could appear was eight $(M=3.36$ appearances, $S D$ $=1.54$ appearances, range $=0-8)$, although participants were not told this.

After each catching attempt, participants immediately estimated the speed of the fish by performing the speed bisection task. They entered their responses ("slow" or "fast") on labeled buttons on the joystick. Using the keyboard, participants then entered the number of times the square had appeared on the fish. No feedback was given on either the speed judgment 
or the square-counting response. Participants completed six blocks. Each block contained 36 trials ( 2 initial fish positions $\times 6$ fish speeds $\times 3$ net heights). Order within a block was randomized.

\section{Results and discussion}

One participant did not understand the task and never released the net. Two participants had multiple data points that were 1.5 times below the interquartile range (one had poor catching performance, and one had poor counting performance). All three were removed prior to the analyses. Perceived speed was assessed by calculating the point of subjective equality (PSE). PSEs were calculated for each participant for each net size condition from binary regressions. Due to complete or partial separation, convergence was not achieved for three participants with the middle net, for one participant for the small net, and for one participant for the big net. SPSS's approximation of the PSE in these cases was still useful, so these data points were included. Eliminating these data points did not change the outcomes. All reported standard errors were calculated within subjects unless otherwise specified.

As a manipulation check, we ensured that fish-catching performance varied as a function of net size. Proportions of fish successfully caught were submitted to a repeated measures analysis of variance (ANOVA) with Net Size as the within-subjects factor. Participants caught the fish more successfully as net size increased, $F(2,46)=1,058.35, p<.001$, $\eta_{\mathrm{p}}{ }^{2}=.98$ (see Fig. 2).

Net size effect on perceived speed As is predicted by an action-specific account of perception, ease to catch the fish

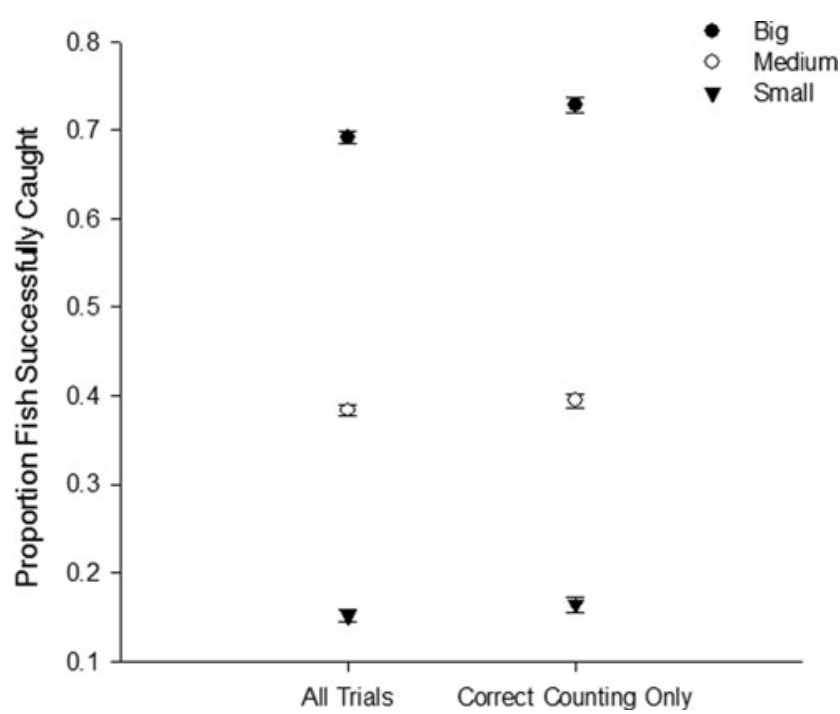

Fig. 2 Proportions of fish successfully caught as a function of net size for Experiment 1. Data on the left are for all trials, and data on the right are only for trials on which squares were counted correctly. Error bars indicate one SEM calculated within subjects influenced the PSEs (see Fig. 3). The PSEs were submitted to a repeated measures ANOVA with Net Size as the withinsubjects factor. This analysis revealed a significant effect of net size on the PSEs, $F(2,46)=36.79, p<.001, \eta_{\mathrm{p}}{ }^{2}=.62$ : Participants judged the fish as moving slower when they used the big net than when they used the small net.

PSEs are a standard measure in psychophysics, but one might argue that the PSEs in this experiment reflect differences in memory rather than differences in perception, because the judgments were made after the fish had stopped moving (Cooper, Sterling, Bacon, \& Bridgeman, 2012). Prior research has demonstrated similar effects, however, when the judgments are made while the target is still moving (Witt \& Sugovic, 2012). In addition, the present paradigm has the advantage that it provides an implicit, action-based measure of perceived speed that is performed while the fish is visibly moving. This measure is known as net release time, or netRT. If the fish is genuinely perceived as moving slower when the net is big, participants should wait longer to release the big net than the small net. Consistent with this prediction, it has been demonstrated that netRTs are slower for the big than for the small net, and that this difference is not attributable to a participant's strategy (Witt \& Sugovic, 2013a).

NetRTs faster than $100 \mathrm{~ms}$ were classified as errors of anticipation and were removed prior to analysis. NetRTs slower than 1.5 times the interquartile range for each fish speed were also removed. Combined, the eliminated trials accounted for $3 \%$ of the data. The remaining trials were summarized by taking the mean netRT for each participant for each net size. These means were submitted to a repeated measures ANOVA with Net Size as the within-subjects factor. Net size

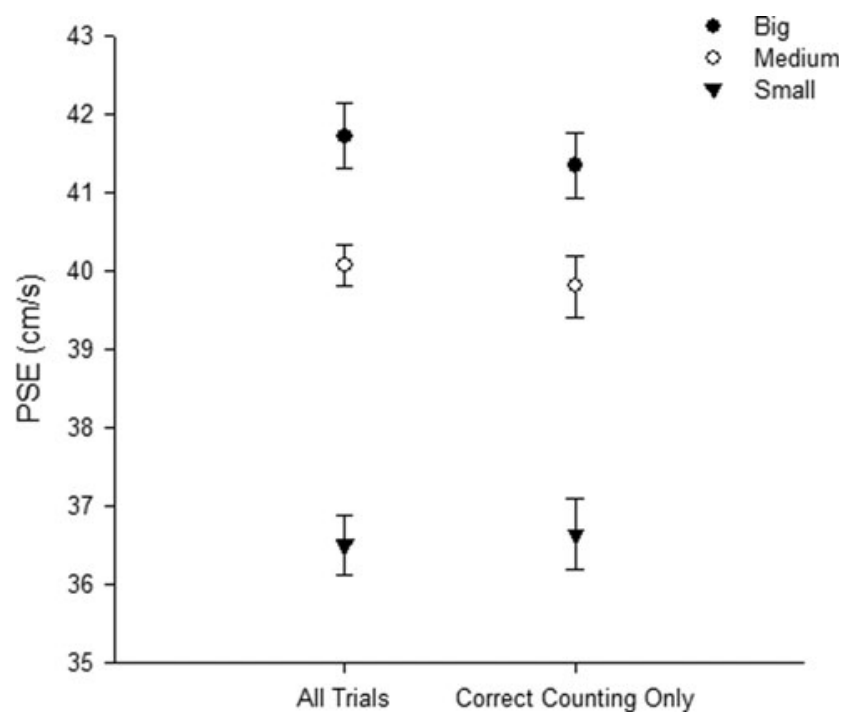

Fig. 3 Points of subjective equality (PSEs) as a function of net size for Experiment 1. A lower PSE indicates the fish was judged as faster. Data on the left are for all trials, and data on the right are only for trials for which squares were counted correctly. Error bars indicate one SEM calculated within subjects 
significantly influenced netRTs, $F(2,46)=4.84, p=.012, \eta_{\mathrm{p}}{ }^{2}$ $=.17$ (see Fig. 4). This replicates previous research and shows that participants wait longer to release the big than the small net. This finding implies that perceivers see the fish as moving slower when they play with the big net, which is why they wait longer.

Both the explicit measure of perceived fish speed (PSEs) and the action-based measure of perceived fish speed (netRT) revealed a significant effect of fish-catching performance. This is consistent with the claim that perception is action-specific, and is in line with numerous previous results. The question of interest, however, was whether attention was the mechanism underlying these effects.

Square counting task The purpose of the square-counting task was to force participants to continuously attend the fish throughout the trial, to ensure that attention was equated across the different net size and fish speed conditions (the task could not be successfully performed in the absence of sustained attention to the fish). The finding that actionspecific effects were observed despite this additional manipulation is evidence against an attention-based account. This issue can be examined further, however, by comparing perceived fish speeds on trials on which the squares were correctly counted. It could be argued that errors on the squarecounting task reflect an inability to maintain attention on the fish and that the action-specific effects observed are primarily attributable to a differential distribution of errors on the square-counting task across net sizes.

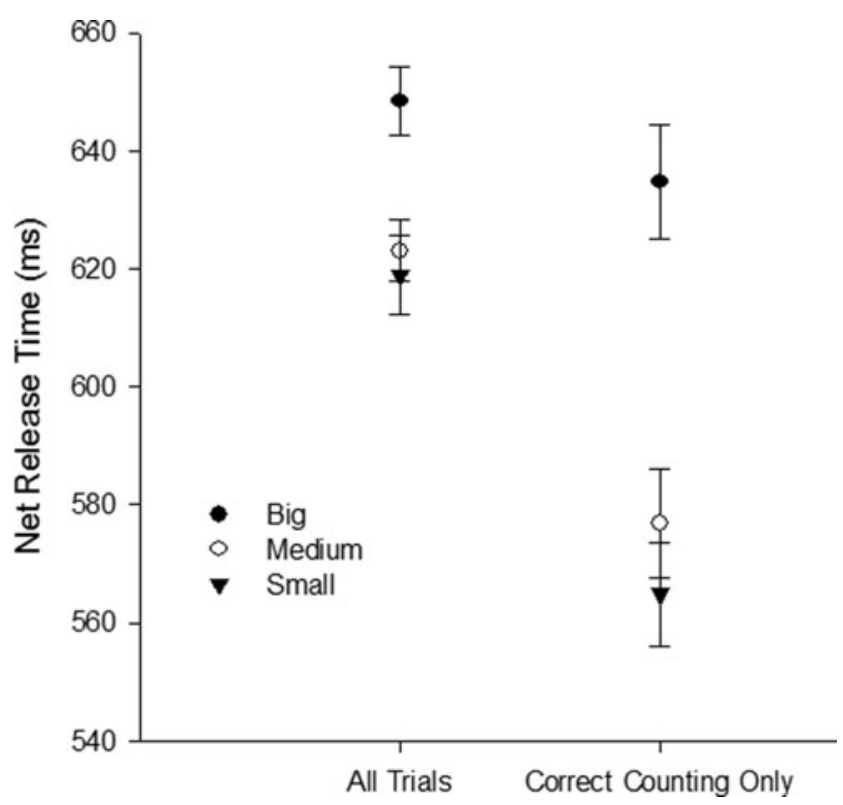

Fig. 4 Net release times (netRTs) as a function of net size for Experiment 1. A higher netRT implies that the fish appeared slower. Data on the left are for all trials, and data on the right are only for trials on which squares were counted correctly. Error bars indicate one SEM calculated within subjects
The mean proportion of trials on which squares were accurately counted was calculated for each participant $(M=.63$, $S D=.14$, range $=.34-.86$; that performance was not substantially higher is indicative of the square-counting task being sufficiently challenging as to require continuous attention). Proportions correct were entered into a repeated measures ANOVA with Net Size as a within-subjects factor. Net size did not significantly influence the proportions of trials on which squares were counted correctly, $F(2,46)=1.72, p=$ $.19, \eta_{\mathrm{p}}{ }^{2}=.07$ (small net: $M=.62$ proportion correct, $S D=.14$; medium net: $M=.63, S D=.14$; big net: $M=.65, S D=.15$ ). This is indicative of attention being equated across our conditions. Nevertheless, to help ensure equality across net sizes, we recomputed the PSEs and netRTs only for trials on which square-counting performance was accurate. Net size still significantly influenced both PSEs, $F(2,46)=21.48, p<.001$, $\eta_{\mathrm{p}}{ }^{2}=.48$, and netRTs, $F(2,46)=10.92, p<.001, \eta_{\mathrm{p}}{ }^{2}=.32$ (see Figs. 3 and 4). This finding verifies that the effect of net size on perceived speed was not driven by attention.

This action-specific effect was unaffected by the squarecounting task, which is inconsistent with an attention-based account. The present data cannot, however, address the issue of whether attention plays some moderating role in the effect of fish-catching performance on perceived fish speed. Some researchers have claimed that attention directed toward the target is necessary for action-specific effects to occur (CañalBruland \& van der Kamp, 2009; Cañal-Bruland et al., 2011), and the present results are consistent with this claim. The purpose of Experiment 2 was to address whether attention toward the target is necessary for net size to influence perceived fish speed.

\section{Experiment 2: stationary fixation}

\section{Method}

We recruited 19 participants who had not participated in Experiment 1 and who were naïve as to the purpose the experiment. A post-hoc power analysis indicated that we achieved over $95 \%$ power with this number of participants to obtain the effect of net size on perceived fish speed.

The procedure was the same as in Experiment 1, except that the squares were white and appeared at the center of the display throughout the experiment (see Fig. 5). Maintaining fixation to count the squares accurately thus required attention to be diverted away from the fish. If attention is critical to action-specific effects, we would expect a reduction in the magnitude of these effects - or their complete eliminationin the present experiment. As before, participants attempted to catch fish with different-sized nets and estimated the speed of the fish. 


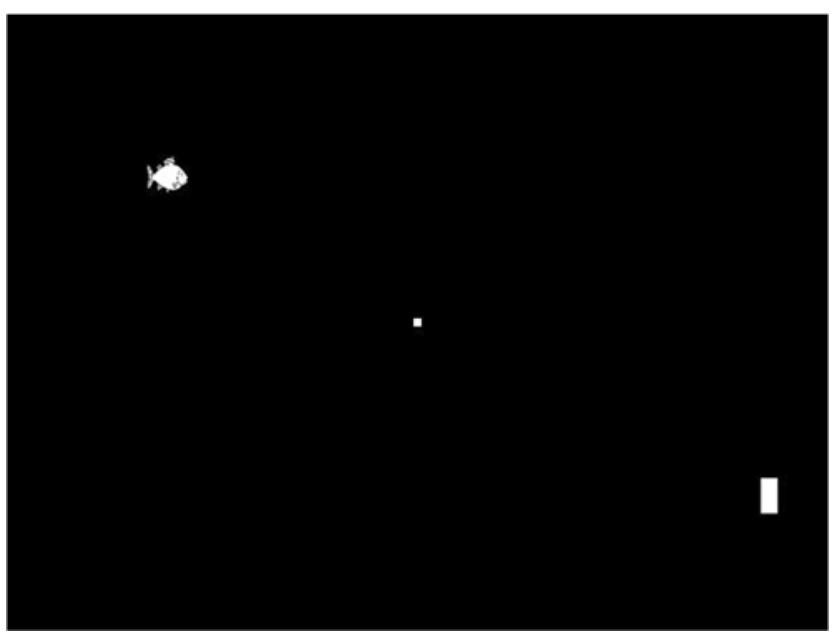

Fig. 5 Example display from Experiment 2: The fish has just started moving, the square is visible, and the net is small and has not yet been released

\section{Results and discussion}

We calculated PSEs and netRTs for each net size for each participant, as before. Two participants were deemed outliers and removed from further analyses. Both had counting accuracy scores 1.5 times lower than the interquartile range, and both had at least one PSE that was 1.5 times greater than the interquartile range.

As expected, net size significantly influenced the proportions of fish successfully caught, $F(2,32)=1,211.41, p<.001$, $\eta_{\mathrm{p}}{ }^{2}=.99$ (see Fig. 6). Net size also marginally influenced square-counting performance, $F(2,32)=3.09, p=.06, \eta_{\mathrm{p}}{ }^{2}=$ .16 , with the linear contrast also being marginally significant, $F(1,16)=3.86, p=.07, \eta_{\mathrm{p}}^{2}=.19$ (small net: $\mathrm{M}=.58$ proportion correct, $\mathrm{SD}=.14$; medium net: $\mathrm{M}=.61, \mathrm{SD}=$ .11 , big net: $\mathrm{M}=.62, \mathrm{SD}=.12$ ). This marginal effect cannot

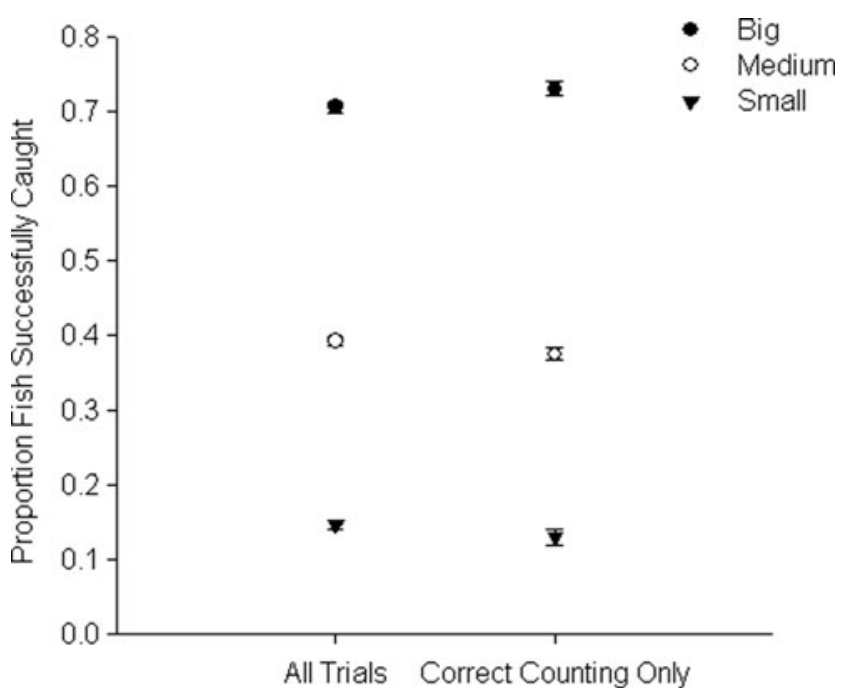

Fig. 6 Proportions of fish successfully caught as a function of net size for Experiment 2. Data on the left are for all trials, and data on the right are only for trials on which squares were counted correctly. Error bars indicate one $S E M$ calculated within subjects account for the action-specific effect, however, because it makes the opposite prediction. According to the AubertFleischl illusion, given that the square required participants to attend away from the fish, the fish should have appeared faster when the net was big than when it was small.

We analyzed the data from all trials and from trials on which squares were counted accurately (70.52\% of trials). Net size influenced the PSEs, $F(2,32)=18.56, p<.001, \eta_{\mathrm{p}}{ }^{2}$ $=.54$, and also the PSEs on correct counting trials, $F(2,32)=$ $14.80, p<.001, \eta_{\mathrm{p}}{ }^{2}=.48$ (see Fig. 7). Even though attention was diverted away from the fish, net size still significantly influenced the PSEs: Participants judged the fish as moving faster when the net was smaller and less effective for catching the fish. Corroborating the finding with explicit speed judgments, netRTs were also influenced by net size, as was shown by analyses with all trials, $F(2,32)=8.39, p=.001, \eta_{\mathrm{p}}{ }^{2}=.34$, and with trials on which squares were counted correctly, $F(2$, 32 ) $=8.10, p=.001, \eta_{\mathrm{p}}{ }^{2}=.34$ (see Fig. 8). Both types of measures suggest that fish that were easier to catch appeared slower, even when attention was diverted elsewhere.

\section{Combined analyses across experiments}

A direct comparison of Experiments 1 and 2 suggests that diverting attention away from the target by placing the squares in the middle of the display did not diminish the effect of net size on apparent speed (see Fig. 9). We computed difference

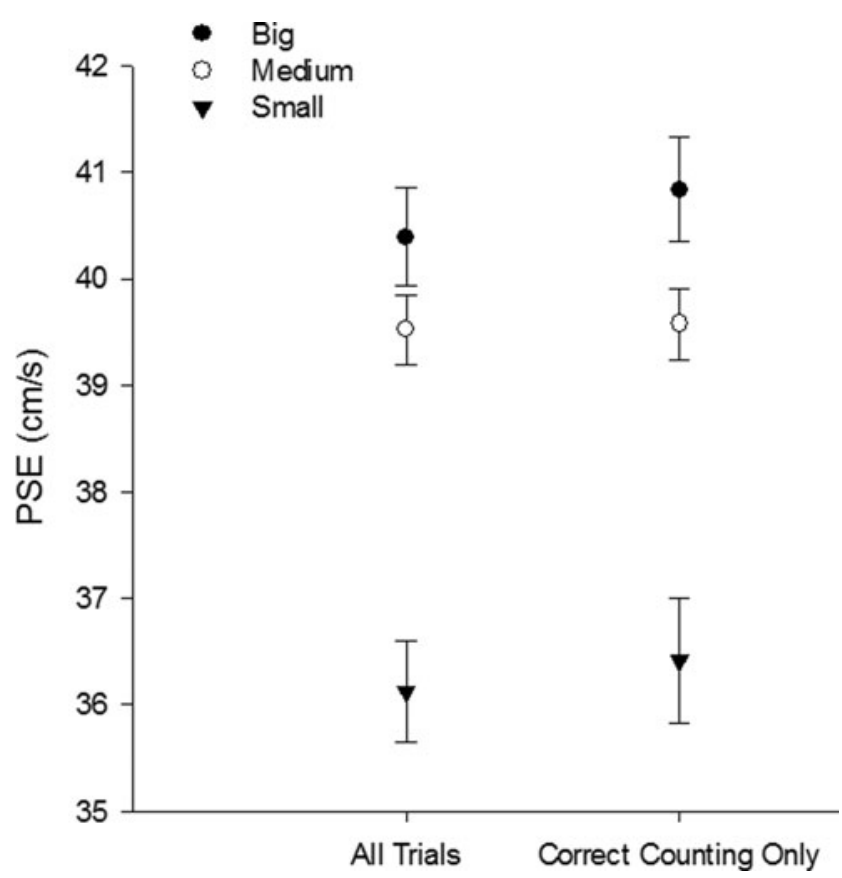

Fig. 7 Points of subjective equality (PSEs) as a function of net size for Experiment 2. A lower PSE represents seeing the fish as moving faster. Data on the left are for all trials, and data on the right are only for trials for which squares were counted correctly. Error bars indicate one SEM calculated within subjects 


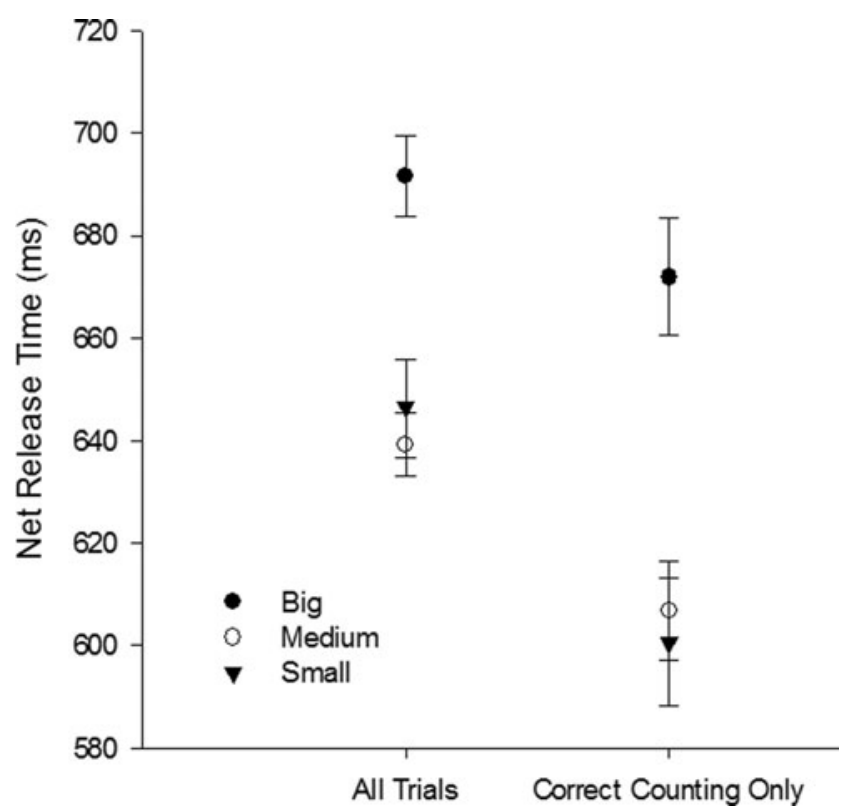

Fig. 8 Net release times (netRTs) as a function of net size for Experiment 2. A higher netRT implies that the fish appeared slower. Data on the left are for all trials, and data on the right are only for trials for which squares were counted correctly. Error bars indicate one SEM calculated within subjects

scores by subtracting the small-net value from the big-net value $^{2}$ for the PSEs and the netRTs for trials on which the squares were correctly counted. These difference score measures were submitted to a multivariate ANOVA with Experiment as a between-subjects factor. For both measures, the intercept was significant [PSEs, $F(1,39)=51.01, p<.001$, $\eta_{\mathrm{p}}{ }^{2}=.57$; netRTs, $\left.F(1,39)=28.23, p<.001, \eta_{\mathrm{p}}{ }^{2}=.42\right]$. An effect in the intercept signals that the difference score for each measure was significantly different from 0 . Experiment was not a significant predictor of either of the difference scores [PSEs, $F(1,39)=0.05, p>.83, \eta_{\mathrm{p}}^{2}<.01$; netRTS, $F(1,39)$ $\left.<0.01, p>.96, \eta_{\mathrm{p}}{ }^{2}<.01\right]$. This lack of effect indicates that the difference scores were not affected by experiment. In other words, directing attention toward versus away from the fish did not impact the effect of net size on perceived fish speed.

Both square-counting tasks required fixation on the square's location in order to successfully count the squares. The square was too small and its appearance too random to allow for successful counting while not maintaining gaze toward the square's location. That said, it is possible that the square-counting task engaged attention in different ways when the square was located on the fish versus at the center of the screen. Indeed, the two versions of the square-counting

\footnotetext{
${ }^{2}$ Conducting a repeated measures ANOVA with Net Size as a withinsubjects factor and Experiment as a between-subjects factor produced the same pattern of significance, with net size being a significant predictor and the interaction between net size and experiment being nonsignificant. Difference scores were used instead to make comparisons of the net effects across experiments easier to visualize.
}

task produced opposite effects on fish-catching performance. The mean proportions of fish successfully caught were entered into a repeated measures ANOVA with Counting Accuracy (correct vs. incorrect) as a within-subjects factor and Experiment as a between-subjects factor. Participants caught the fish more successfully in Experiment 1, when attention was drawn to the fish, than in Experiment 2, when attention was drawn away from it, $F(1,39)=5.06, p<.05, \eta_{\mathrm{p}}{ }^{2}=.12$. This makes intuitive sense, given that the square appeared on the fish in Experiment 1, so participants had one less object to attend in that experiment than did the participants in Experiment 2. The effect of counting accuracy was not significant, $F<1$, but the interaction between experiment and counting accuracy was, $F(1,39)=13.61, p=.001, \eta_{\mathrm{p}}{ }^{2}=.26$. In Experiment 1, participants caught the fish more successfully when they counted correctly $(M=.43$ proportion of fish successfully caught, $S D=$ $.06)$ than when they counted incorrectly $(M=.37, S D=.06)$, $t(23)=3.14, p<.01, d_{\mathrm{RM}}=0.99$. In contrast, in Experiment 2, participants caught the fish more successfully when they counted incorrectly $(M=.45, S D=.08)$ than when they counted correctly $(M=.41, S D=.04), t(16)=2.25, p<.05, d_{\mathrm{RM}}=$ 0.61 . This pattern of results is consistent with the idea that the two square-counting tasks engaged attention in different ways. However, given that the effects of net size on perceived speed were similar across the two experiments, the results actually bolster the argument that attention is not involved in this particular action-specific effect. In addition, examination of the effect sizes previously obtained with the fishing task when there was no secondary task revealed similar effect sizes to those obtained here (explicit judgments, $\eta_{\mathrm{p}}{ }^{2}=.43$; netRTs, $\eta_{\mathrm{p}}{ }^{2}$ $=.31$; Witt \& Sugovic, 2013a). Not only did equating or diverting attention fail to eliminate the effect of net size on perceived speed, but equating or diverting attention did not do much, if anything, to reduce this action-specific effect.

It is also of interest to examine how the fish-catching performance on each trial impacted the perceived fish speed. Previously collected data revealed mixed results, such that sometimes the target appeared significantly slower after a successful catch than after a miss, but many times no effects of catching success on perceived speed have emerged (Witt \& Sugovic, 2010, 2013a). Calculating PSEs for each net size and trial outcome (successful catch vs. miss) is problematic, because there are so few successes with the small net that convergence cannot be achieved. Consequently, we analyzed the raw response data using a hierarchical linear model (HLM 7). The dependent variable was the response on each trial $(0=$ slow, $1=$ fast ), which was modeled using a Bernoulli distribution. With experiment as a predictor, we found no significant effects, no significant interactions, and no change to the significance or parameter estimates for any of the other factors. As such, we collapsed our data across experiments. The subsequent predictors were trial success $(0=$ miss, $1=h i t)$, square-counting accuracy $(0=$ incorrect, $1=$ correct $)$, fish 


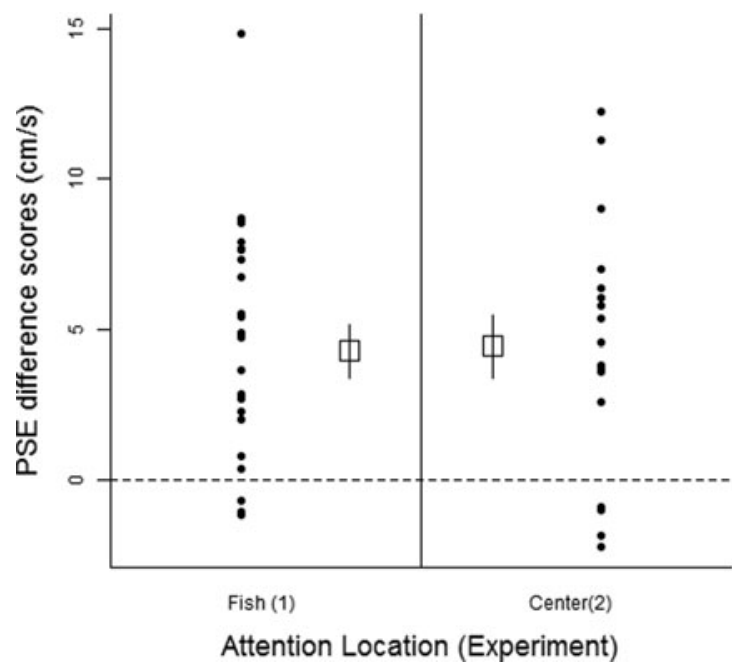

Fig. 9 Difference scores for PSEs (left) and netRTs (right) for trials on which squares were counted correctly are plotted as a function of experiment. Difference scores were calculated as the value with the big net minus the value with the small net. Difference scores show the effect of net size on perceived fish speed. A higher value indicates that net size

speed (centered around the group mean), net size $(-1=$ small, $0=$ medium, $1=$ big $)$, and the interaction between trial success and net size. The reported findings are results from the population-average model with robust standard errors (see Fig. 10). Net size significantly influenced estimated speed $(t$ $=-4.74, p<.001, \beta=-.38$ ), and trial success also significantly influenced estimated speed $(t=-2.22, p=.03, \beta=-.24)$. The coefficients reveal that net size had a larger impact on estimated speed than did trial success. The interaction between net size and trial success was not significant $(t=0.29, p>.77, \beta=$ $.03)$. Fish speed influenced reported speed $(t=20.17, p<.001$,

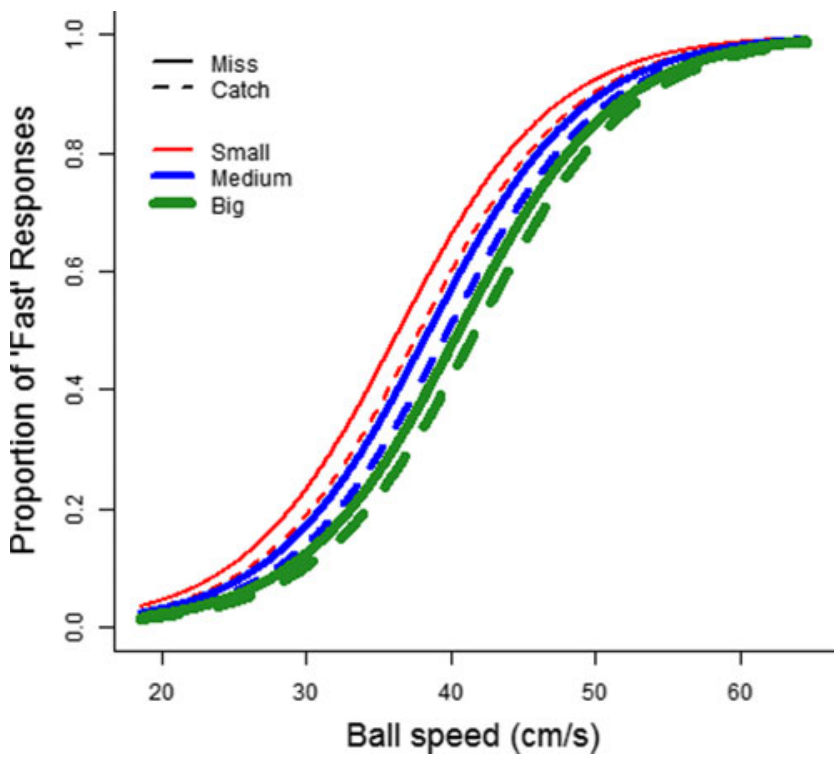

Fig. 10 Proportions of "fast" responses as a function of ball speed, trial success, and net size. The data are combined across both experiments. Curve estimates are from the hierarchical linear model results. See the text for details

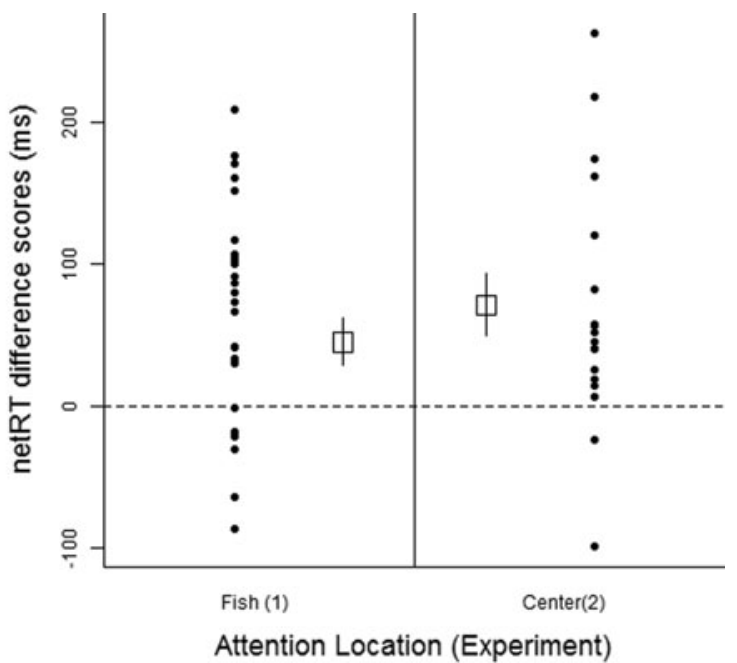

had a larger effect on perceived fish speed in the predicted direction. The dotted lines at 0 represent the points at which net size would have no effect on perceived speed. Small circles represent the data from each participant, and large squares represent the group means. Error bars indicate one $S E M$ calculated between subjects

$\beta=.18)$, but square-counting accuracy did not significantly influence reported speed $(t=1.06, p=.29, \beta=.10)$. These results show that although participants were more likely to label fish that had been missed as moving faster, this did not account for the effect of net size on perceived fish speed.

\section{General discussion}

According to the action-specific account of perception, people perceive the spatial layout of the environment in relation to their abilities to act in it (Witt, 2011). For example, softballs look bigger to people who are hitting better than others (Gray, 2013; Witt \& Proffitt, 2005). What remains unknown is the mechanism by which a person's ability to act influences perception. One possible mechanism is that the ease to perform a task changes how a person attends to the target, and that differences in attention are responsible for differences in perception (e.g., Gray et al., 2014). Attention as the underlying mechanism has some appeal, because most theories of spatial perception can account for differences in how an object looks as the result of changes in the inputs or weights (Philbeck \& Witt, 2015). However, given our results showing that attention is not an underlying mechanism, the implication is that action directly influences perception, such that information about action is directly integrated with or processed along with optical cues.

Action-specific effects have been criticized as to whether they reveal genuine influences on perception, but no gold standards exist for how to assess the evidence (see Philbeck \& Witt, 2015, for a review). Recently, a framework has offered six potential pitfalls that can be used to evaluate the perceptual 
nature of an effect (Firestone \& Scholl, 2015); an effect that falls into any of the six pitfalls is discounted as being a genuinely perceptual effect. The six pitfalls include (1) having an overly confirmatory research agenda, (2) effects due to an influence on judgments rather than on perception, (3) effects due to response biases or task demands, (4) effects due to lowlevel differences in optical information, (5) effects due to differences in attention, and (6) effects on memory rather than perception. Prior research has ruled out five of the six pitfalls as they relate to this particular action-specific effect (Witt \& Sugovic, 2012, 2013a, b; for a review, see Witt et al., 2015). The one remaining pitfall that could discredit this effect as being perceptual related to attention. Thus, the present experiments were critical, because they ruled out the final, previously unexplored pitfall, relating to attention rather than to a direct influence of perception. We can now conclude that, at least in this case, action genuinely influences perception.

We examined the potential role of attention for actionspecific effects by having participants perform a secondary task that required them to continuously attend a target fish (Exp. 1) or to continuously divert attention away from the target fish (Exp. 2). This afforded us an opportunity to test three potential accounts for action-specific effects: attentionbased, perceptual accentuation, and non-attention-based. Attention is a plausible mechanism for a variety of reasons, with the most important being that looking behavior can influence the perceived speed of an object. During smooth pursuit of a moving object, the object appears to move slower than when a nearby stationary point is fixated instead. This phenomenon is known as the Aubert-Fleischl illusion (Aubert, 1886; Fleischl, 1882). In the context of action-specific effects, this effect of looking behavior on apparent speed could account for the effect of catching ease on the perceived speed of a fish. According to this account, when playing with the big net, participants would be more likely to focus on the fish, which explains why the fish appears slower than when playing with the small net.

If action-specific effects are attributable to an underlying attentional mechanism, then according to the attention-based (Gray et al., 2014) and perceptual accentuation (CañalBruland et al., 2011) accounts, we would have expected our secondary square-counting task to lead to an elimination of these effects or, at the very least, a reduction in their magnitude. This was not the case. Action-specific effects were observed in the present study, independent of whether attention was directed toward or diverted away from the target fish, including on trials on which the square-counting task was performed correctly (implying that attention was engaged by the secondary task throughout the trial). Moreover, the magnitude of the observed effects were similar to those in a previous study using the same paradigm, albeit in the absence of the secondary task (Witt \& Sugovic, 2013a).
That this action-specific effect was unaffected by our secondary task is strong evidence against a critical role for attention. This is particularly the case given that our secondary square-counting task required that attention be divided between the square/fish and the net (Exp. 1) and between the fish, the net, and the square at the center of the screen (Exp. 2). There have been numerous demonstrations that dividing attention often leads to performance deficits across a variety of tasks and modalities (Anderson \& Craik, 1974; Bonnel \& Hafter, 1998; Bonnel \& Prinzmetal, 1998; Craik, Govoni, Naveh-Benjamin, \& Anderson, 1996; Folk, 2010; Salvucci \& Taatgen, 2008; Strayer \& Drews, 2007), given humans' limited cognitive capacity. As such, had we observed a reduction or elimination of action-specific effects, it would have required additional investigation to determine whether this was attributable to attention per se or to a general capacity limit. The absence of any influence of an attentional manipulation on action-specific effects suggests that the underlying mechanism was unrelated to attention.

The present study represents one of the first attempts to directly measure the impact of attention on action-specific effects (see also Cañal-Bruland et al., 2011; van der Hoort \& Ehrsson, 2014) by directly manipulating whether participants were required to continuously attend-or divert attention away from - a target object within the context of a dual-task paradigm. Previous investigations of this issue have often examined the question in an indirect manner, in the absence of an attentional manipulation. Previously, we found that objects that are easier to catch appear to be moving slower (Witt et al., 2014; Witt \& Sugovic, 2010, 2012, 2013a, b, c; Witt et al., 2012). In the present experiments, a secondary task forced participants to continuously attend to the fish (or continuously attend elsewhere), thereby equating attention across net sizes. The results demonstrate that action-specific effects are observed independent of how attention is oriented. These studies rule out a role for overt attention in this particular action-specific effect, and suggest that a perceiver's ability to catch a moving object directly influences the perceived speed of the object.

Author note This research was supported by grants from the National Science Foundation (Nos. BCS-0957051, BCS-1314162, and BCS1348916) to J.K.W.

\section{References}

Anderson, C. M., \& Craik, F. I. M. (1974). The effect of a concurrent task on recall from primary memory. Journal of Verbal Learning and Verbal Behavior, 13, 103-113.

Aubert, H. (1886). Die Bewegungsempfindung [The sensation of movement]. Pflügers Archiv, 39, 347-370.

Bloesch, E. K., Davoli, C. C., Roth, N., Brockmole, J. R., \& Abrams, R. A. (2012). Watch this! Observed tool use affects perceived distance. 
Psychonomic Bulletin \& Review, 19, 177-183. doi:10.3758/s13423011-0200-Z

Bonnel, A.-M., \& Hafter, E. (1998). Divided attention between simultaneous auditory and visual signals. Perception \& Psychophysics, 60, 179-190.

Bonnel, A.-M., \& Prinzmetal, W. (1998). Dividing attention between the color and the shape of objects. Perception \& Psychophysics, 60, $113-124$

Cañal-Bruland, R., Pijpers, J. R., \& Oudejans, R. R. (2010). The influence of anxiety on action-specific perception. Anxiety, Stress, and Coping, 23, 353-361.

Cañal-Bruland, R., \& van der Kamp, J. (2009). Action goals influences action-specific perception. Psychonomic Bulletin \& Review, 16, 1100-1105. doi:10.3758/PBR.16.6.1100

Cañal-Bruland, R., Zhu, F. F., van der Kamp, J., \& Masters, R. S. W. (2011). Target-directed visual attention is a prerequisite for actionspecific perception. Acta Psychologica, 136, 285-289.

Cooper, A. D., Sterling, C. P., Bacon, M. P., \& Bridgeman, B. (2012). Does action affect perception or memory? Vision Research, 62, 235240. doi:10.1016/j.visres.2012.04.009

Craik, F. I. M., Govoni, R., Naveh-Benjamin, M., \& Anderson, N. D. (1996). The effects of divided attention on encoding and retrieval processes in human memory. Journal of Experimental Psychology: General, 125, 159-180. doi:10.1037/0096-3445.125.2.159

Doerrfeld, A., Sebanz, N., \& Shiffrar, M. (2012). Expecting to lift a box together makes the load look lighter. Psychological Research, 76, 467-475. doi:10.1007/s00426-011-0398-4

Firestone, C., \& Scholl, B. J. (2015). Cognition does not affect perception: Evaluating the evidence for "top-down" effects. Behavioral and Brain Sciences. doi:10.1017/S0140525X15000965

Fleischl, E. V. (1882). Physiologisch-optische Notizen, 2. Mittheilung [Physiologically optical notes, second notification]. Sitzungsberichte der Akademie der Wissenschaften, 86, 7-25.

Folk, C. L. (2010). Attention: Divided. In E. B. Goldstein (Ed.), Encyclopedia of perception (pp. 84-87). Thousand Oaks: Sage.

Gibson, J. J. (1979). The ecological approach to visual perception. Boston: Houghton Mifflin.

Gray, R. (2013). Being selective at the plate: Processing dependence between perceptual variables relates to hitting goals and performance. Journal of Experimental Psychology: Human Perception and Performance, 39, 1124-1142.

Gray, R., Navia, J. A., \& Allsop, J. (2014). Action-specific effects in aviation: What determines judged runway size? Perception, 43, $145-154$.

Hayhoe, M., \& Ballard, D. (2005). Eye movements in natural behavior. Trends in Cognitive Sciences, 9, 188-194. doi:10.1016/j.tics.2005. 02.009

Johnston, E. B., Cumming, B. G., \& Landy, M. S. (1995). Integration of stereopsis and motion shape cues. Vision Research, 34, 2259-2275.

Lee, Y., Lee, S., Carello, C., \& Turvey, M. T. (2012). An archer's perceived form scales the "hitableness" of archery targets. Journal of Experimental Psychology: Human Perception and Performance, $38,1125-1131$

Philbeck, J. W., \& Witt, J. K. (2015). Action-specific influences on perception and postperceptual processes: Present controversies and future directions. Psychological Bulletin, 141, 1120-1144. doi:10. 1037/a0039738

Salvucci, D. D., \& Taatgen, N. A. (2008). Threaded cognition: An integrated theory of concurrent multitasking. Psychological Review, $115,101-130$.

Strayer, D. L., \& Drews, F. A. (2007). Multitasking in the automobile. In A. F. Kramer, D. A. Weiegmann, \& A. Kirlik (Eds.), Attention: From theory to practice (pp. 121-133). New York: Oxford University Press.

Taylor, J. E. T., Witt, J. K., \& Sugovic, M. (2011). When walls are no longer barriers: Perception of wall height in parkour. Perception, 40, 757-760. doi:10.1068/P6855

van der Hoort, B., \& Ehrsson, H. H. (2014). Body ownership affects visual perception of object size by rescaling the visual representation of external space. Attention, Perception, \& Psychophysics, 76, 1414-1428. doi:10.3758/s13414-014-0664-9

Vickers, J. N. (1992). Gaze control in putting. Perception, 21, 117-132.

Witt, J. K. (2011). Action's effect on perception. Current Directions in Psychological Science, 20, 201-206. doi:10.1177/ 0963721411408770

Witt, J. K., \& Proffitt, D. R. (2005). See the ball, hit the ball—Apparent ball size is correlated with batting average. Psychological Science, 16, 937-938. doi:10.1111/j.1467-9280.2005.01640.x

Witt, J. K., \& Riley, M. A. (2014). Discovering your inner Gibson: Reconciling action-specific and ecological approaches to perception-action. Psychonomic Bulletin \& Review, 21, 1353-1370. doi: 10.3758/s13423-014-0623-4

Witt, J. K., South, S. C., \& Sugovic, M. (2014). A perceiver's own abilities influence perception, even when observing others. Psychonomic Bulletin \& Review, 21, 384-389.

Witt, J. K., \& Sugovic, M. (2010). Performance and ease influence perceived speed. Perception, 39, 1341-1353. doi:10.1068/P6699

Witt, J. K., \& Sugovic, M. (2012). Does ease to block a ball affect perceived ball speed? Examination of alternative hypotheses. Journal of Experimental Psychology: Human Perception and Performance, 38, 1202-1214. doi:10.1037/a0026512

Witt, J. K., \& Sugovic, M. (2013a). Catching ease influences perceived speed: Evidence for action-specific effects from action-based measures. Psychonomic Bulletin \& Review, 20, 1364-1370. doi:10. 3758/s13423-013-0448-6

Witt, J. K., \& Sugovic, M. (2013b). Response bias cannot explain actionspecific effects: Evidence from compliant and non-compliant participants. Perception, 42, 138-152. doi:10.1068/p7367

Witt, J. K., \& Sugovic, M. (2013c). Spiders appear to move faster than non-threatening objects regardless of one's ability to block them. Acta Psychologica, 143, 284-291. doi:10.1016/j.actpsy. 2013.04.011

Witt, J. K., Sugovic, M., Tenhundfeld, N. T., \& King, Z. R. (2015). An action-specific effect on perception that avoids all pitfalls. Behavioral and Brain Sciences.

Witt, J. K., Sugovic, M., \& Taylor, J. E. T. (2012). Action-specific effects in a social context: Others' abilities influence perceived speed. Journal of Experimental Psychology: Human Perception and Performance, 38, 715-725. doi:10.1037/a0026261 